\title{
A note on evaluations of some exponential sums
}

\author{
by \\ MARKo J. Moisio (Vaasa)
}

1. Introduction. The recent article [1] gives explicit evaluations for exponential sums of the form

$$
S\left(a, p^{\alpha}+1\right):=\sum_{x \in \mathbb{F}_{q}} \chi\left(a x^{p^{\alpha}+1}\right)
$$

where $\chi$ is a non-trivial additive character of the finite field $\mathbb{F}_{q}, q=p^{e}$ odd, and $a \in \mathbb{F}_{q}^{*}$. In my dissertation [5], in particular in [4], I considered more generally the sums $S(a, N)$ for all factors $N$ of $p^{\alpha}+1$. The aim of the present note is to evaluate $S(a, N)$ in a short way, following [4]. We note that our result is also valid for even $q$, and the technique used in our proof can also be used to evaluate certain sums of the form

$$
\sum_{x \in \mathbb{F}_{q}} \chi\left(a x^{p^{\alpha}+1}+b x\right)
$$

2. Evaluation of $S(a, N)$. Let $\mathbb{F}_{q}$ denote the finite field with $q=p^{e}$ elements, $\chi_{1}$ the canonical additive character of $\mathbb{F}_{q}$ and $\alpha$ a non-negative integer. Let $N$ be an arbitrary divisor of $p^{\alpha}+1$. Our task is to evaluate the sums

$$
S(a, N):=\sum_{x \in \mathbb{F}_{q}} \chi_{1}\left(a x^{N}\right)
$$

for non-zero elements $a$ of $\mathbb{F}_{q}$.

Let $d=\operatorname{gcd}(\alpha, e)$. Since $S(a, N)=S\left(a, \operatorname{gcd}\left(N, p^{e}-1\right)\right)$ and

$$
\operatorname{gcd}\left(p^{\alpha}+1, p^{e}-1\right)= \begin{cases}1 & \text { if } e / d \text { is odd and } p=2, \\ 2 & \text { if } e / d \text { is odd and } p>2, \\ p^{d}+1 & \text { if } e / d \text { is even }\end{cases}
$$

as proved in [1] and [3, p. 175], it is enough to consider sums $S(a, n)$ for all divisors $n$ of $p^{d}+1$. The case $e / d$ odd is easily established (see [1]).

2000 Mathematics Subject Classification: Primary 11T24. 
To state our result we fix a primitive element of $\mathbb{F}_{q}$, say $\gamma$, and denote the multiplicative group of $\mathbb{F}_{q}$ by $\mathbb{F}_{q}^{*}$.

Theorem 1. Let $e=2 s d$ and $n \mid p^{d}+1$. Then

$$
\sum_{x \in \mathbb{F}_{q}} \chi_{1}\left(a x^{n}\right)= \begin{cases}(-1)^{s} p^{s d} & \text { if } \operatorname{ind}_{\gamma} a \neq k(\bmod n), \\ (-1)^{s-1}(n-1) p^{s d} & \text { if } \operatorname{ind}_{\gamma} a \equiv k(\bmod n),\end{cases}
$$

where $k=0$ if

(A) $p=2$; or $p>2$ and $2 \mid s ;$ or $p>2,2 \nmid s$ and $2 \mid\left(p^{d}+1\right) / n$,

and $k=n / 2$ if

(B) $p>2,2 \nmid s$ and $2 \nmid\left(p^{d}+1\right) / n$.

In the special case $n=p^{d}+1, p$ odd, our Theorem 1 gives Theorem 2 of $[1]$.

The proof of our theorem is based on the relation (see [2, p. 217])

$$
\sum_{x \in \mathbb{F}_{q}^{*}} \chi_{1}\left(a x^{n}\right)=\sum_{\psi \in H} G(\bar{\psi}) \psi(a)
$$

where $H$ is the subgroup of order $n$ of the multiplicative character group of $\mathbb{F}_{q}$, and $G(\bar{\psi})$ is the Gauss sum

$$
G(\bar{\psi})=\sum_{x \in \mathbb{F}_{q}^{*}} \chi_{1}(x) \bar{\psi}(x) .
$$

Proof of Theorem 1. Let $H^{\prime}$ be the subgroup of order $n$ of the multiplicative character group of $\mathbb{F}_{p^{2 d}}$. The surjectivity of the norm mapping $\mathrm{N}$ from $\mathbb{F}_{q}$ to $\mathbb{F}_{p^{2 d}}$ implies $H=\left\{\psi \circ \mathrm{N} \mid \psi \in H^{\prime}\right\}$. Now (1) and the Davenport-Hasse theorem (see [2, pp. 195-199]) imply

(2) $\sum_{x \in \mathbb{F}_{q}^{*}} \chi_{1}\left(a x^{n}\right)=\sum_{\psi \in H^{\prime}} G(\bar{\psi} \circ \mathrm{N}) \psi(\mathrm{N}(a))=(-1)^{s-1} \sum_{\psi \in H^{\prime}} G^{\prime}(\bar{\psi})^{s} \psi(\mathrm{N}(a))$,

where $G^{\prime}(\bar{\psi})$ is computed over $\mathbb{F}_{p^{2 d}}$.

Let $\psi_{0}$ denote the trivial multiplicative character of $\mathbb{F}_{p^{2 d}}$. Since $G^{\prime}\left(\psi_{0}\right)=$ -1 , it follows from (2) that

$$
\sum_{x \in \mathbb{F}_{q}} \chi_{1}\left(a x^{n}\right)=(-1)^{s-1} \sum_{\psi \in H^{\prime *}} G^{\prime}(\bar{\psi})^{s} \psi(\mathrm{N}(a)),
$$

where $H^{\prime *}:=H^{\prime} \backslash\left\{\psi_{0}\right\}$.

Let $\psi \in H^{\prime *}$. Since ord $(\psi) \mid p^{d}+1$, we observe that Stickelberger's theorem (see [2, p. 202]) is applicable.

Now, if $p=2$ or $2 \mid s$, then $G^{\prime}(\bar{\psi})^{s}=p^{s d}$. To consider the remaining cases, we fix a generator of the multiplicative character group of $\mathbb{F}_{p^{2 d}}$, say $\lambda$, and define $t=\left(p^{2 d}-1\right) / n$. 
Now $\psi=\lambda^{t j}$ for some $j \in\{1, \ldots, n-1\}$. Since $\operatorname{ord}(\psi)=n / \operatorname{gcd}(n, j)$, we see that $\left(p^{d}+1\right) / \operatorname{ord}(\psi)$ is even if $\left(p^{d}+1\right) / n$ is even. Consequently, $G^{\prime}(\bar{\psi})^{s}=p^{s d}$ if $\left(p^{d}+1\right) / n$ is even.

Thus in Case A we have

$$
\sum_{x \in \mathbb{F}_{q}} \chi_{1}\left(a x^{n}\right)=(-1)^{s-1} p^{s d} \sum_{j=1}^{n-1} \lambda^{t j}(\mathrm{~N}(a)) .
$$

In Case B, $\left(p^{d}+1\right) / \operatorname{ord}(\psi)$ is even if and only if $j$ is even. Thus

$$
\sum_{x \in \mathbb{F}_{q}} \chi_{1}\left(a x^{n}\right)=(-1)^{s-1} p^{s d} \sum_{j=1}^{n-1}(-1)^{j} \lambda^{t j}(\mathrm{~N}(a)) .
$$

Noting that $\mathrm{N}(\gamma)$ is a primitive element of $\mathbb{F}_{p^{2 d}}$, we easily obtain the result.

If $n=p^{d}+1$ and $s=1$, for example, we can prove by a more or less similar reasoning (see [5])

Theorem 2. Let $a, b \in \mathbb{F}_{q}, b \neq 0$. Then

$$
\sum_{x \in \mathbb{F}_{q}} \chi_{1}\left(a x^{p^{d}+1}+b x\right)= \begin{cases}0 & \text { if } a+a^{p^{d}}=0, \\ -p^{d} \chi_{1}^{\prime}\left(-b^{p^{d}+1}\left(a+a^{p^{d}}\right)^{-1}\right) & \text { if } a+a^{p^{d}} \neq 0,\end{cases}
$$

where $\chi_{1}^{\prime}$ is the canonical additive character of the field $\mathbb{F}_{p^{d}}$.

Acknowledgements. The author is indebted to Professor Keijo Väänänen for his valuable comments on a preliminary version of this note.

\section{References}

[1] R. S. Coulter, Explicit evaluations of some Weil sums, Acta Arith. 83 (1998), 241-251.

[2] R. Lidl and H. Niederreiter, Finite Fields, Encyclopedia Math. Appl. 20, Addison-Wesley, Reading, 1983 (now distributed by Cambridge Univ. Press).

[3] R. J. McEliece, Finite Fields for Computer Scientists and Engineers, Kluwer, Dordrecht, 1987.

[4] M. J. Moisio, On relations between certain exponential sums and multiple Kloosterman sums and some applications to coding theory, preprint, 1997.

[5] - Exponential sums, Gauss sums and cyclic codes, Dissertation, Acta Univ. Oul. A 306, 1998.

Department of Mathematics and Statistics

University of Vaasa

Box 700, 65101 Vaasa, Finland

E-mail:mamo@uwasa.fi 